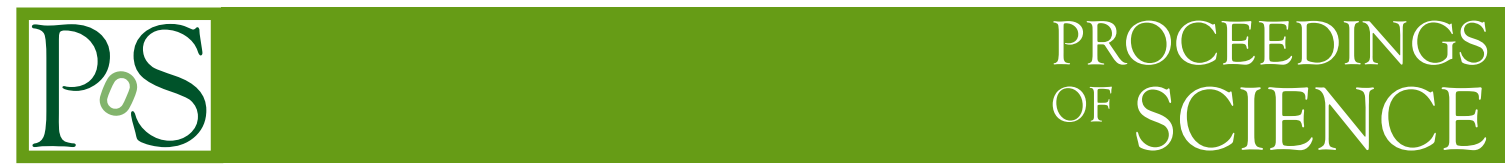

\title{
Compressed Baryonic Matter
}

\section{Subhasis Chattopadhyay, Subikash Choudhury, Debojit Sarkar}

Variable Energy Cyclotron Centre, 1/AF, Bidhan Nagar, Kolkata 700 064, W.B., India E-mail:

subevecc.gov.in

Strongly interacting matter under extremely high net-baryon density (several times the nuclear matter density) and relatively low temperature will be created in the nuclear collisions of beam energy in the range of 10 - $45 \mathrm{AGeV}$. In the phase-diagram of the strongly interacting matter, the region of high net-baryon density and low temperature contains several major landmarks like the Critical End Point (CEP), Quarkyonic matter, color superconducting phase among others. Present theoretical understanding of this region of phase-diagram, experimental observables, status of the present and future experiments engaged in exploring the high density matter have been discussed.

7th International Conference on Physics and Astrophysics of Quark Gluon Plasma

1-5 February, 2015

Kolkata, India 


\section{Introduction}

High temperature quark-gluon plasma (QGP) is formed in central heavy ion collisions at topRHIC and LHC energies. According to lattice QCD calculations, the transition is a cross-over suggesting a continuous change from one phase to the other. The scenario is represented by the topleft corner of the T- $\mu_{B}$ (Fig. 1) phase diagram of the strongly interacting matter. The conjectured phase diagram at lower temperature and higher net-baryon density exhibits several substructures e.g., the critical end point (CEP), Quarkyonic phase of matter, Color Flavour Locked (CFL) phase, color superconducting phase as shown in Fig. $1[1,2]$. Both the existence and the locations of these landmarks are topics of extensive theoretical study. It is therefore of extreme importance that explorations are made to study these regions experimentally.

Lowering of collision energy in high energy heavy ion collisions will lead the colliding nuclei to overlap each other with only partial transparency thereby creating a system of lower temperature and of higher net-baryon density. The resultant net-baryon density may exceed nuclear saturation density by a large factor representing a system of higher baryon-chemical potential. It should be noted that such collisions are likely to create matter at high density but not of zero temperature. Various models and assumed equations of state are used to describe the system and to estimate the expected thermodynamic variables like $\mathrm{T}, \mu_{B}$.

All the landmarks predicted to be present at high density correspond to model calculations of the properties of strongly interacting matter. We discuss them briefly here,

- CEP: In the phase diagram, the cross-over transition discovered at RHIC and LHC at high temperature and almost zero net-baryon density should be separated from the $1^{\text {st }}$ order phase transition at lower temperature and higher $\mu_{B}$ by a Critical End Point (CEP) as has been observed in the phase digram of water as shown in Fig. 2 (left) [4]. Beyond the CEP, watervapour transforms smoothly to liquid water. The phase-diagram of Fig. 1 has also been redrawn like the phase-diagram of water (T,P) and different exotic phases and CEP have been shown $[3,5]$.

- Quarkyonic Phase

This phase has been conjectured to exist in-between the deconfined pure QGP state and the confined hadronic state. The conjectured baryon-rich phase is believed to be confined, but chirally symmetric. Even though several theoretical calculations have been performed, however, no clear predictions are given about the signatures of this phase [6].

The predicted rich structures at high $\mu_{B}$ and low temperature led experimentalists to setup experiments to explore these exotic phenomena. These experiments require heavy ion collisions at beam energies significantly below top RHIC energies. In our discussions, collisions at an energy below the top RHIC energy is referred to as the low enery collisions. The facilities where such experiments are being performed and in the planning phase include (a) Beam Energy Scan at RHIC and SPS (b) Compressed baryonic Matter (CBM) experiment at the upcoming FAIR facility and (c) the proposed Multi-Purpose Detector (MPD) at the upcoming NICA facility at Dubna, Russia.

The high energy heavy ion collision experiments have evolved from $\mathrm{E}_{l a b}$ of about $1 \mathrm{AGeV}$ at LBNL to $\sqrt{s}=2.76 \mathrm{TeV}$ at LHC. The experiments performed in early days measured the most 


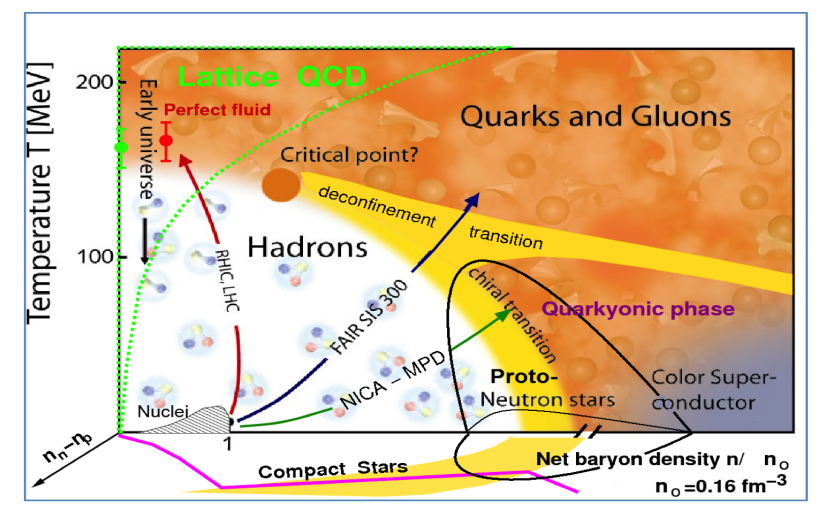

Figure 1: Phase-diagram of the strongly interacting matter in $\left(\mathrm{T}, \mathrm{n}_{B}\right)$ plane.
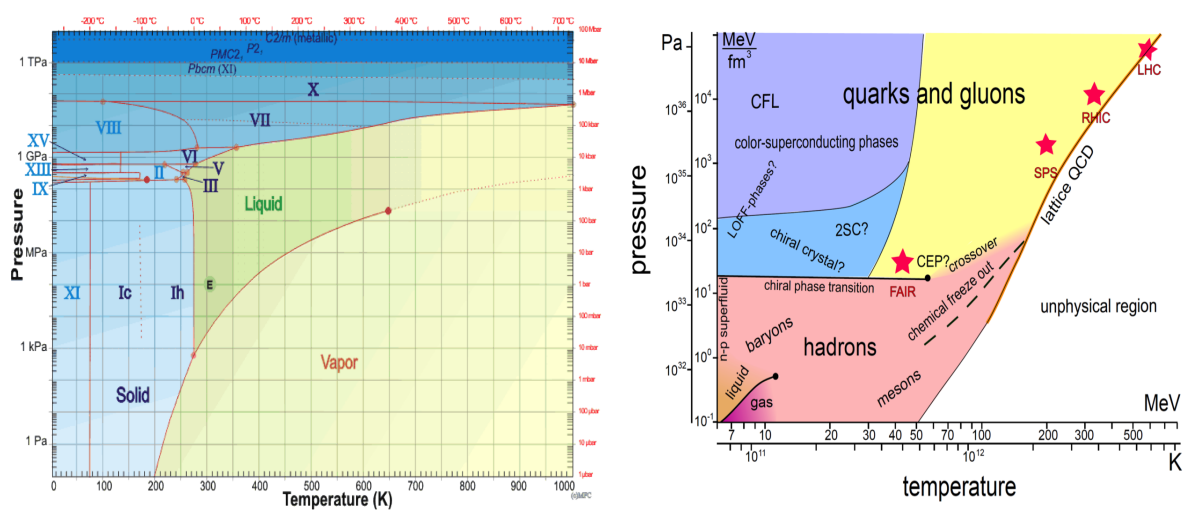

Figure 2: (left) (T-P) phase-diagram of water (right) corresponding (T-P) constructed phase diagram of the strongly interacting matter.

abunduntly produced particles like proton, kaon, pion and discovered collective flow. In the ongoing experiments like the Beam energy scan at RHIC, scans are being performed over a broad range of collision energy and on a wide range of observables. Upcoming experiments like CBM are focussed to measure rare probes which could not be measured up to date like $\mathrm{J} / \psi$ at threshold energies. The data collected by STAR and PHENIX experiments at RHIC and SHINE/NA49 at SPS have contributed significantly in improving the knowledge of the high density matter created in low energy collisions.

We divide our discussions in the following sections, the next section discusses the theoretical robustness of various predicted substructures at high $\mu_{B}$ and the accessibility by experiments. In section-3, we discuss the major observables having potential to explore the high density medium. We go over the main findings at RHIC and SPS low energy programme in section-4. Upcoming experiments, their main features and timelines will be discussed in section-5 with a summary in section-6. 


\section{Physics at high net-baryon density}

In low-energy nuclear collisions, the nuclei are likely to overlap each other, generating high net-baryon density and forming a partonic medium. Unfortunately, the procedure of simulation of statistical QCD in lattice can not be applied to the strongly interacting system at high $\mu_{B}$ due to the well-known sign problem [7]. Efforts have been made to overcome the issue by several approaches like Taylor series expansion of the potentials [8]. In absence of not-so-reliable results from the lattice calculations, effective models like NJL model with the inclusion of Polyakov loop for modeling the deconfinement (PNJL) are being used to understand the system at high density [9]. The phase-diagrams developed at the FAIR energy regime using these models mostly contains these landmarks like CEP, first-order phase transition and superconducting phase. A common observation is that the location of CEPs predicted by lattice and effective models differ. The netbaryon density likely to be achieved in these collisions has been estimated using transport models like UrQMD, HSD. The thermodynamical parameters like $\mathrm{T}, \mu_{B}$ describing the system are extracted using the statistical models. According to transport models, the system created in the low energy collisions reaches a density upto 6 times the nuclear saturation density even at $\mathrm{E}_{\text {lab }}=10 \mathrm{AGeV}$., at which it is hard to assume the existence of a hadronic phase.

If we start at the high temperature region of the phase diagram and proceed towards a cooler system at higher $\mu_{B}$, a changeover from cross-over to a first order phase transition is predicted by many models. This prediction includes the existence of the CEP which actually is the most prominent hunting issue in high density matter. However, efforts are also being made to focus on the $1^{\text {st }}$ order transition line because the discovery of the first order transition line is equivalent to the discovery of the CEP. Model calculations speculate, that the region that lies in-between the deconfined phase-transition line at the top and the chiral phase-transition line at the bottom is a quarkyonic phase which is a baryon-rich confined but chirally symmetric phase [6]. Beyond this region, we arrive at a dense region comparable to the core of neutron star. As can be seen from the previous discussions that the missing theory predictions for the high-density region clearly calls for experimental data.

The estimation of density can be obtained using the transport models like UrQMD, HSD and others as shown in the Fig. 3(left). It is clear that the peak density is reached at the time of interpenetration of the nuclei and lives longer at lower energy. At higher collision energy, the density reached is higher but shorter-lived. The observables carrying the message of high density therefore need to survive the space-time evolution of the system till freezeout. We also show in Fig. 3 (right) the evolution of the system in the $\left(\mathrm{T}, \mu_{B}\right)$ plane as has been modelled by UrQMD. The freezeout curve is shown from a thermal model calculations [10]. The locations of CEPs as predicted by the lattice QCD are also shown in the figure [11]. It is seen that even the Au-Au collisions at $\mathrm{E}_{l a b}=10$ $\mathrm{AGeV}$ is an interesting place for studying both the phase transition and the CEP.

One question that has drawn significant attention for designing an experiment is whether a deconfined medium will be produced at low energy collisions like $\mathrm{E}_{l a b}=10 \mathrm{~A} \mathrm{GeV}$ to be available at SIS-100 of FAIR. Calculations on the structure of neutron stars predict that the core of the neutron-star at a density of several times the nuclear saturation density might be a deconfined state of quark matter. However, the duration of the highest density state in heavy ion collisions is a few $\mathrm{fm} / \mathrm{c}$ as compared to the neutron star which is a long-lived object. In case of neutron-star, 

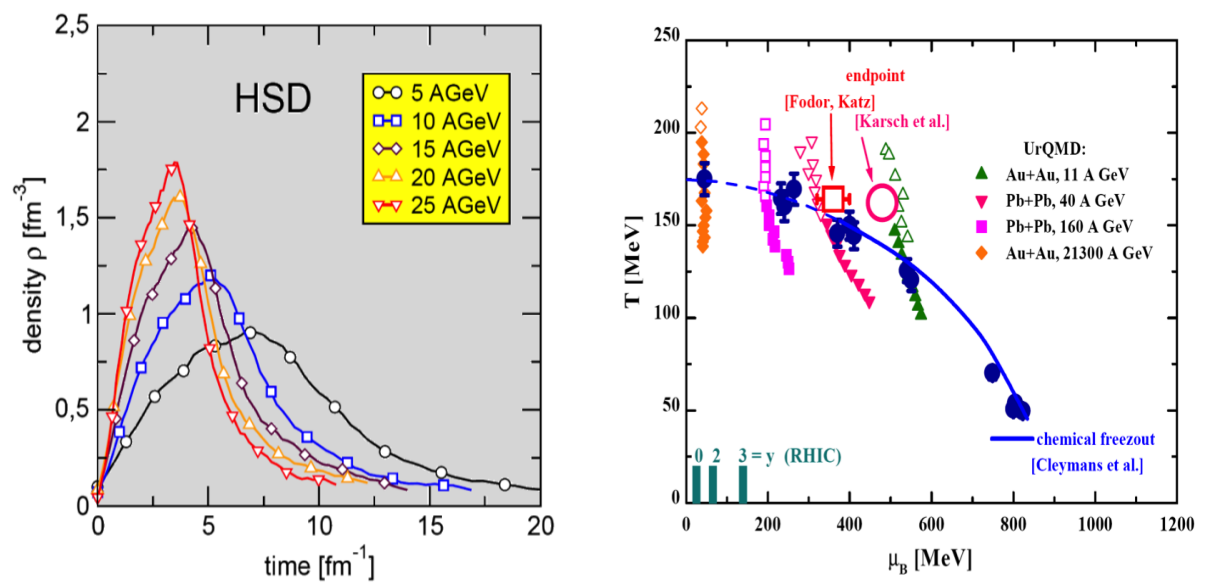

Figure 3: (left) Evolution of net-baryon densiy in Au+Au collisions with time at different $\mathrm{E}_{l a b}$ (right) time evolution of temperature-baryochemical potential profile as obtained from UrQMD, the T- $\mu_{B}$ freezeout curve as obtained from Thermal model is also shown.

conclusions about the compositon of the core are concluded from observations like cooling pattern, in case of heavy ion collisions, however, more direct observables have been predicted to exist. The motivation of the study of heavy ion collisions from neutron star has therefore its own limitations.

\section{Observables}

Most of the wide-ranging observables being used in high energy heavy ion collision experiments studying the high temperature domain are expected to be valid at high density study as well. We here discuss only a few of these observables that describe specific aspects of the system.

\subsection{Equation of State (EOS) of the medium}

Spectra of the produced particles are used commonly to model the EOS of the medium. The space-time evolution of the system created in such collisions assume the medium to be equilibrated at a given time after the collision, the equilibration time is approximately of the order of the time required for the colliding nuclei to pass each other. With lowering of the centre of mass energy of the collisions, the equilibration time also increases. The observables like transverse momentum $\left(\mathrm{p}_{T}\right)$ spectra of the produced particles, the coefficients of the azimuthal asymmetries of the produced particles $\left(\mathrm{v}_{2}, \mathrm{v}_{3}\right.$ and higher order coefficients), their mass dependences are the observables that are compared with the space-time evolution models of the system for various equations of state. The EOS that matches well with the results are said to represent the system. The equilibration of the system is therefore not directly observed, but is validated by the observations that the models using an equilibrated system like hydrodynamical expansion match data quite well [12].

In case of low energy heavy ion collisions, in addition to the procedure discussed above, because of the creation of high density, the constituents undergo interactions and create newer particles like strange baryons. The yields of strange baryons are therefore sensitive to the density achieved in the medium. The results can therefore be used to extract the EOS of the medium. 


\subsection{Mass modifications of vector mesons}

In a high density medium, one process that is responsible for melting of the masses of the vector mesons is the chiral symmetry restoration [13]. There are models invoking other mechanisms also can explain melting. The short lifetime of $\rho$-mesons results in their decay inside the medium and thus $\rho$ meson is a suitable candidate to be a messanger of the interaction with the medium. In addition to that, dileptons, the decay products of the vector mesons will not interact with the medium on its passage and will carry unhindered information of $\rho$ inside the medium. It is therefore a useful tool to study the effect of density in the generation of mass in a high density medium. Even though, the connection between the chiral symmetry restoration and the melting of $\rho$ is not direct, however, conclusions can be made in a model-dependent way.

\subsection{Fluctuations}

One of the most promising candidates that is anticipated to be discovered at high $\mu_{B}$ collision is the CEP. Thermodynamically, such a critical phenomenon is characterized by the discontinuity in susceptibility of the medium to external signals. In case of heavy ion collisions, it has been argued that the event by event fluctuations of the yields of the conserved quantities like net-charge, netstrangeness, net-baryons among others are representatives of the susceptibility of the medium, i.e. response of the system to external forces [14]. At CEP, the correlation length of the order parameter diverge resulting in a divergence of the fluctuations. The Fluctuations are usually measured by the variance of the distribution of the measured quantity, as it is related to the correlation length $(\zeta)$ of the medium. It has also been shown by the lattice calculations that the higher order moments like skewness and Kurtosis of the distributions are more sensitive to the divergence of the correlation length as the dependence is of higher exponents in case of higher moments. It is therefore suggested that the excitation function of these moments or their combination might show non-monotonic behaviour depicting the occurance of the critical phenomena.

\subsection{Enhancement of strangeness}

Enhancement of the production of strage hadrons in AA collisions has been predicted to be a signature for the creation of the partonic medium almost from the begining of this field of research. The reason has been attributed to the generation of more strange quarks in a partonic medium where the levels of light flavours are completely filled. There are hadronic channels through which strange particles might also be generated. It has been seen in a model-dependent way that the strangeness enhancement factor measured by the ratio of yields of strange particles in AA collisions to those in the pp collisions, increases fastest in the SIS100 energy range. The effect is more prominent in case of multi-strange hadrons like $\Omega$. It has been seen that the models with partonic degrees of freedom gives significantly higher anti-strange particle yield as compared to the hadronic version of the model. It has also been shown that at lower energy collisions, because of the enhancement of strangeness production and the production of nucleons, the yields of hypernuclei increase significantly.

\section{Results from low energy experiments so far}

Two programmes have been undertaken to scan the collision energy and search for the onset 
of phase-transition and/or the CEP. SHINE at SPS-CERN, the successor of NA49 experiment has performed scanning in the beam-target combinations and of beam energy. The matrix covered by SHINE is shown in the Fig. 4.

The other beam energy scan programme has been undertaken at RHIC in which data have been taken at $\sqrt{s}$ of $200 \mathrm{GeV}, 130 \mathrm{GeV}, 62.4 \mathrm{GeV}, 39 \mathrm{GeV}, 27 \mathrm{GeV}, 19.6 \mathrm{GeV}, 14.5 \mathrm{GeV}, 11.5 \mathrm{GeV}$ and $7.7 \mathrm{GeV}$. The second phase of the programme is scheduled to take place in 2018 to cover upto 5.5 $\mathrm{GeV}$ and a set of fixed target collisions covering to a very low $\sqrt{s}$.

NA49 and SHINE together have reported several major findings that could be explained by phase transition. The observations commonly known as 'horn', and 'step' as shown in Fig. 5 can be explained by models invoking phase transition. In 'horn', $\mathrm{k} / \pi$ ratio shows a peak at around $\sqrt{s}=$ $7 \mathrm{GeV}$, in the 'step', shown on the right panel of the figure, temperature as obtained from the slope of the kaon spectra rises with $\sqrt{s}$, reaches a saturation and then rises again, charateristic of the $1^{s t}$ order phase transition [15].

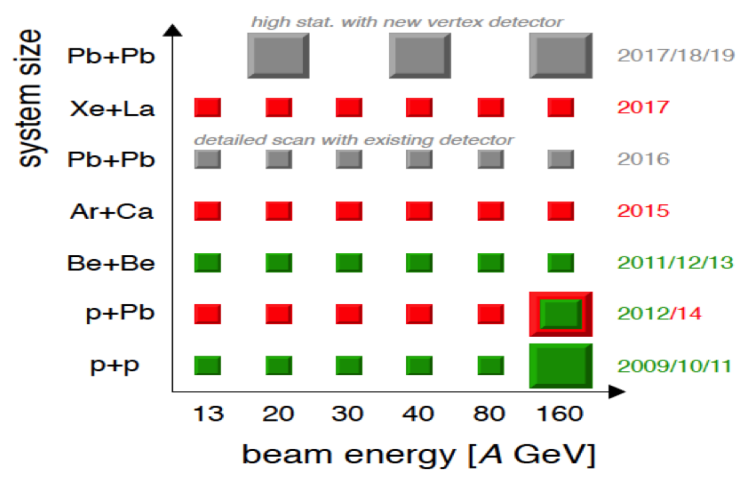

Figure 4: BES setups of the SHINE experiment
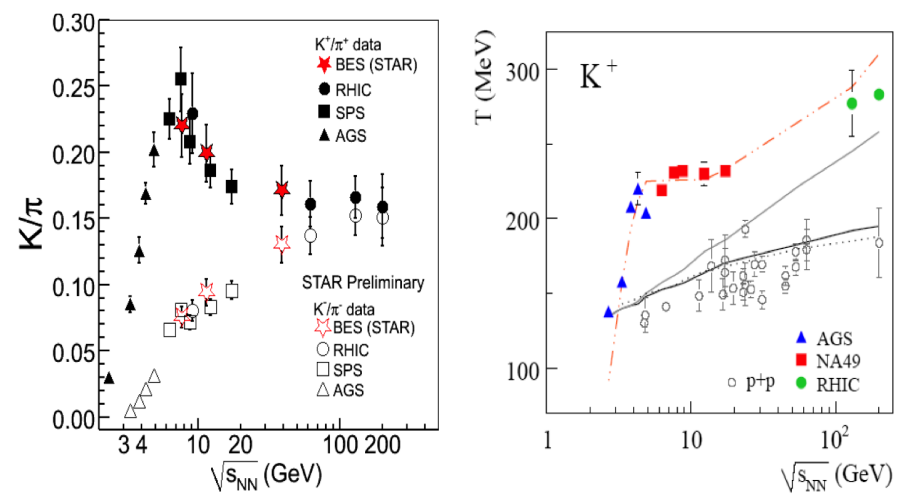

Figure 5: (left) Horn in the k/ $\pi$ ratio, (right) step found in the NA49 experiment. These results can be explained only with the invocation of phase transtion.

At RHIC-BES, main focus was to study the excitation functions till a very low $\sqrt{s}$ of all the observables that led to the conclusion of the creation of the strongly coupled QGP at top RHIC 
energy. STAR has found evidences two of which are shown in Fig. 6. The signals showing crossover transition at top RHIC energy change with decreasing collision energy in such a way that the hadronic matter starts playing major role below $\sqrt{s}$ of $20 \mathrm{GeV}$. However, the explanations of the observations require the modeling of constituents and their interactions of the high density medium properly. In Fig. 6 (left), $\mathrm{R}_{C P}$, representing jet-quenching in a partonic medium at higher $\sqrt{s}$, goes above unity showing no-quenching. In Fig. 6(right), the NCQ scaling of $\mathrm{v}_{2}$ is seen to break down as shown by different $\mathrm{v}_{2}$ values for proton and anti-proton at lower $\sqrt{s}$ [16]. The sensitivity of the
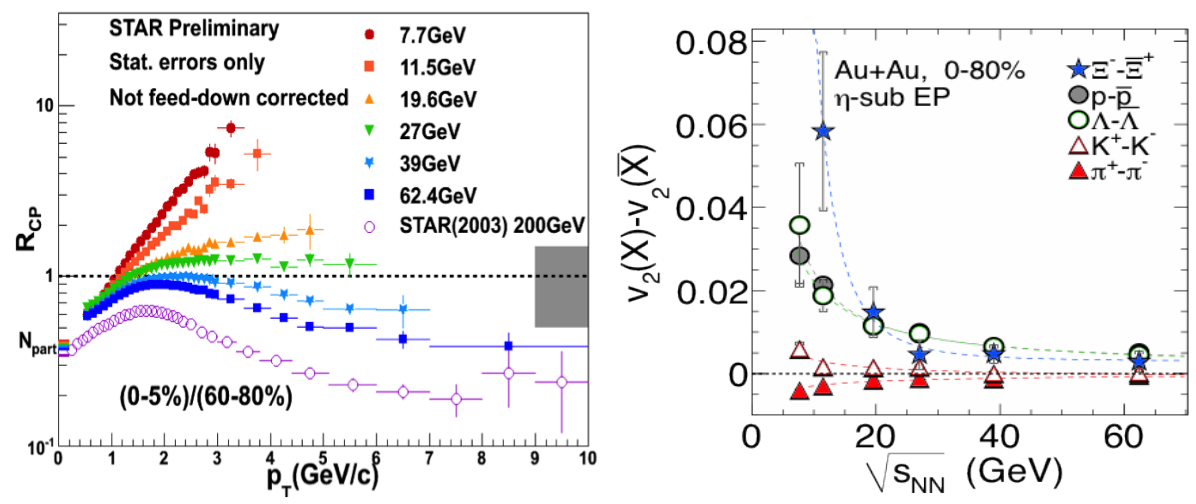

Figure 6: Results from STAR Beam energy scan programme. (Left) $\mathrm{R}_{C P}$ and (right) breaking of NCQ scaling below $\sqrt{s}=39 \mathrm{GeV}$.

observables to the high temperature and low/zero net-baryon density medium need to be evaluated in view of their modifications at high-density, low temperature phase. The deconfined phase at high density will be quark dominated as opposed to the gluon-dominated high temeperature phase. The hadronization and therefore the final observables might be modified accordingly.

One set of observations that have drawn maximum attention are the so-called "higher moments" analysis to study the fluctuations of the conserved quantities like net-charge, net-baryon number. The fluctuations of the conserved quantities in a limited phase space are related to the susceptibility i.e., the response to external impulse and in effect is sensitive to the phase transition and more importantly to CEP. The results from STAR (Fig. 7, top) showing the dependence of $\kappa \sigma^{2}$ ( $\sigma$ and $\kappa$ being the second and fourth moments of the distributions) of net-proton shows an interesting behaviour with $\sqrt{s}$ and is suggested to show a non-monotonic behaviour with an indication of a dip at around $19 \mathrm{GeV}$ [16]. The results are compared with ref. [17], which predicts such a structure for the CEP. STAR is gearing up for detailed study using BES-II by 2018.

\section{Some details of the upcoming high density experiments}

The challenges in low energy heavy ion collision experiments can be illustrated in Fig. 5 in which multiplicity $\times$ Branching ratios of different particles as generated by HSD for central Au $+\mathrm{Au}$ collisions, at $\mathrm{E}_{l a b}$ of $25 \mathrm{AGeV}$ are shown. It is clear that, even though the bulk observables like pions are copiously produced, the rate of production of hadrons with heavier flavours like strange or charm are reduced drastically, thereby requiring higher interaction rate for achieving reasonable statistics. In colliders, even after cooling of beams to improve the luminosity, it is 


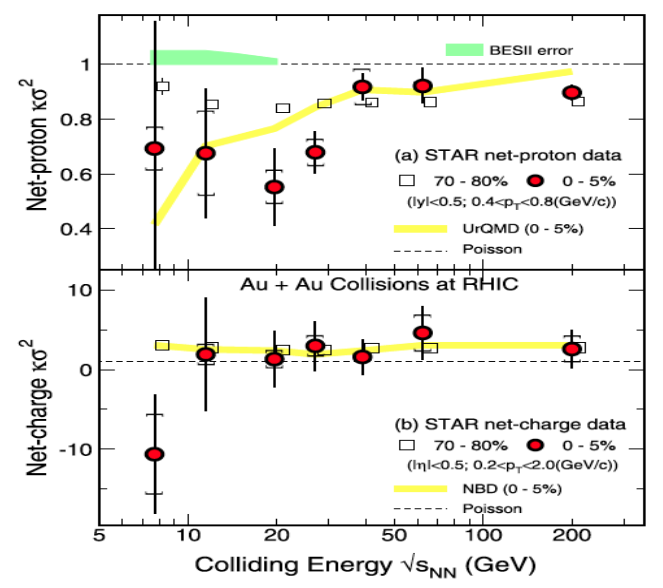

Figure 7: $\kappa \sigma^{2}$ with $\sqrt{s}$ for net-proton (top) and net-charge (bottom) distributions in the BES-I of RHIC.

almost impossible to collect reasonable statistics for rare probes like $\mathrm{J} / \psi$. It is therefore usual practice that fixed target experiments are setup to attain good event statistics in a reasonable data taking period. By increasing the beam intensity and using not-so-thick target, one can enhance the interaction-rate to achieve higher statistics. Higher interaction rates, however, put bigger challenges on the rate capability of the detector technologies.

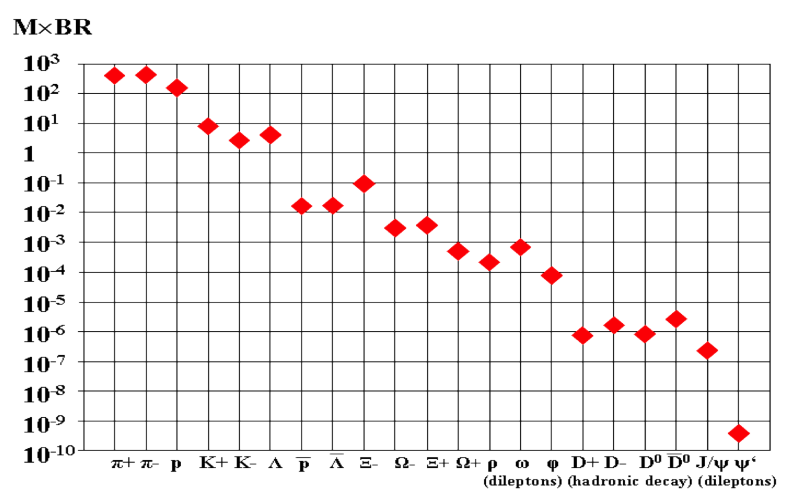

Figure 8: Multiplicity $\times$ Branching ratio for various particles in central $\mathrm{Au}+\mathrm{Au}$ collisions at $\mathrm{E}_{l a b}$ of 25 $\mathrm{AGeV}$

Fig. 9 shows a list of running and upcoming high-density experiments that are aimed to explore the high density matter at relatively lower energy heavy ion collisions. As can be seen from the figure that the $\sqrt{s_{N N}}$ range explored by these experiments cover from $2.7 \mathrm{GeV}$ to $200 \mathrm{GeV}$. At low $\sqrt{s}$, the production cross-sections of the heavier particles reduce drastically and therefore the experiments focussed to detect them are to be operated at very high reaction rates. The experiments mostly to be run in fixed-target mode are to be designed to cope $\approx \mathrm{MHz}$ interaction rate and correspondingly very high radiation level.

One should however take a note of a major difference between the acceptance of particles in collider and fixed-target experiments at different collision energies. In case of collider, the Y- $p_{T}$ 


\begin{tabular}{|l|l|c|}
\hline Experiment & $\begin{array}{l}\text { Energy range } \\
(\text { Au/Pb beams) }\end{array}$ & $\begin{array}{c}\text { Reaction rates } \\
\mathrm{Hz}\end{array}$ \\
\hline $\begin{array}{l}\text { STAR@RHIC } \\
\text { BNL }\end{array}$ & $\sqrt{\mathrm{S}_{\mathrm{NN}}=7-200 \mathrm{GeV}}$ & $\begin{array}{c}1-800 \\
\text { (limitation by luminosity) }\end{array}$ \\
\hline $\begin{array}{l}\text { NA61@SPS } \\
\text { CERN }\end{array}$ & $\begin{array}{l}\mathrm{E}_{\mathrm{kin}}=20-160 \mathrm{~A} \mathrm{GeV} \\
\sqrt{\mathrm{s}_{\mathrm{NN}}}=6.4-17.4 \mathrm{GeV}\end{array}$ & $\begin{array}{c}80 \\
\text { (limitation by detector) }\end{array}$ \\
\hline $\begin{array}{l}\text { MPD@NICA } \\
\text { Dubna }\end{array}$ & $\sqrt{\mathrm{s}_{\mathrm{NN}}=4.0-11.0 \mathrm{GeV}}$ & $\begin{array}{c}\sim 1000 \\
\text { (design luminosity of } \\
10^{27} \mathrm{~cm}^{-2} \mathrm{~s}^{-1} \text { for heayy ions) }\end{array}$ \\
\hline $\begin{array}{l}\text { CBM@FAIR } \\
\text { Darmstadt }\end{array}$ & $\begin{array}{l}\mathrm{E}_{\mathrm{kin}}=2.0-35 \mathrm{~A} \mathrm{GeV} \\
\sqrt{\mathrm{s}_{\mathrm{NN}}}=2.7-8.3 \mathrm{GeV}\end{array}$ & $\begin{array}{c}10^{5}-10^{7} \\
\text { (limitation by detector) }\end{array}$ \\
\hline
\end{tabular}

Figure 9: List of running and upcoming high density experiments and their parameters.

acceptance for pion, proton and kaons remain almost same at different $\sqrt{s}$. However, in case of a fixed target experiment, like NA49 experiment at CERN, the acceptance is highly dependent on the beam energy. In the later case therefore, it is of extreme importance that proper corrections are to be made for any bias that might be introduced due to differences in acceptances.

One experiment named Compressed Baryonic Matter (CBM) at FAIR that is scheduled to take data in 2022 in its first phase of operation is shown in Fig. 10 [18]. The setup consists of a dipole magent with $\pm 25^{\circ}$ opening and a Silicon tracking Station (STS) for tracking. Outside the maget, the experiment can operate in two modes, in the dielectron mode, magnet will be followed by RICH, TOF and TRD detectors, In the dimoun mode, a muon detection system with instrumented absorber will be used. A Projectile spectator detector (PSD) will be used for centrality determination. This new generation experiment alongwith the capability of the measurement of most abundantly produced particles like pion, kaon will also be able to detect rarely produced particles like charmonia and open charm. The experiment will also be equipped with tracking detectors having good particle identification capability so that exotic strange objects can be measured with high statistics. Advanced detectors, self-triggerred readout electronics coupled to high beam intensity from FAIR accelerator will allow CBM to study the details of the high-density matter making it suitable to reach a stage to discover exotic phases. The other upcoming experiments like MPD@NICA is also well-placed to study the high density matter.

\section{Summary}

Following our contemporary understanding of the QCD phase diagram, the region of high $\mu_{B}$ and low temperature contains features that must be explored experimentally. This region can only be accessed using collision energies significantly lower than the top RHIC energy. Several experiments are being conducted and also in planned stage to perform beam energy scan to access important landmarks. Results from RHIC BES-I have indicated some interesting results and high rate experiments like CBM is in the planning stage to focus on detecting the rare probes. The high density-low temeprature region of the phase diagram has become increasingly more intersting in this field of study. 


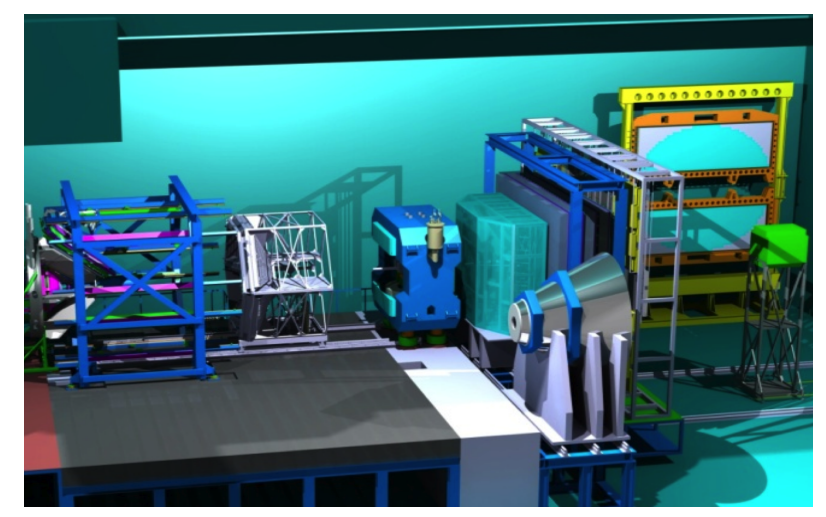

Figure 10: Setup of the Compressed baryonic Matter (CBM) experiment.

\section{References}

[1] B. Friman, C. HÃúhne, J. Knoll, S. Leupold, J. Randrup, R. Rapp, P. Senger (editors) Series: Lecture Notes in Physics, Vol. 814 (2011) 960 p., Hardcover ISBN: 978-3-642-13292-6

[2] Quark Matter series of conferences

[3] McLerran, L. Nucl.Phys.Proc.Suppl. 195 (2009) 275-280 arXiv:0906.2651 [hep-ph]

[4] Chaplin, M., 2007, www.lsbu.ac.uk/water/phase.html

[5] http://arxiv.org/pdf/0801.4256v2.pdf

[6] L. McLerran, R. Pisarski, Nucl. Phys. A 796 (2007) 83; Y. Hidaka, L.D. McLerran, R.D. Pisarski, Nucl. Phys. A 808 (2008) 117

[7] J. Bloch and T. Wettig, JHEP 05 (2011) 048

[8] O. Philipsen, hep-lat/0708.1293v1

[9] K. Yamazaki, T. Matsui / Nuclear Physics A 913 (2013) 19âĂŞ50,Costa, Pedro et al. Europhys.Lett. 86 (2009) 31001 arXiv:0801.3616

[10] S. Wheaton, J. Cleymans and M. Hauer, Comput. Phys. Commun. 180 (2009) 84-106

[11] Z.Fodor, S. D. Katz, arXiv:hep-lat/0402006v1,

[12] P. F. Kolb, U. Heinz arXiv:nucl-th/0305084; K. J. Eskola, H. Niemi, R. Paatelainen, arXiv:1509.02767

[13] R. Rapp, arXiv:1210.3660, P. M. Hohler, R. Rapp: arXiv:1311.2921

[14] M. Doring and V. Koch, arXiv:nucl-th/0204009v1

[15] Rustamov, Anar Central Eur.J.Phys. 10 (2012) 1267-1270 arXiv:1201.4520 [nucl-ex]

[16] Nu Xu, Nuclear Physics A 931 (2014) 1âĂŞ12

[17] M.A. Stephanov, Phys. Rev. Lett. 107, 052301 (2011), J. Phys. G: 38, 124147 (2011)

[18] J. M. Heuser, arXiv:0907.2136 